\title{
Breast Radiotherapy with Mixed Energy Photons; a Model for Optimal Beam Weighting
}

\author{
Mohammadjavad Tahmasebi Birgani ${ }^{1}$, Jafar Fatahiasl ${ }^{1 *}$, Seyed Mohammad \\ Hosseini $^{2}$, Ali Bagheri ${ }^{2}$, Mohammad Ali Behrooz ${ }^{1}$, Mansour Zabiehzadeh ${ }^{1}$, Reza \\ meskani $^{1}$, Maryam Talaei Gomari ${ }^{1}$
}

\begin{abstract}
Utilization of high energy photons (>10MV) with an optimal weight using a mixed energy technique is a practical way to generate a homogenous dose distribution while maintaining adequate target coverage in intact breast radiotherapy. This study represents a model for estimation of this optimal weight for day to day clinical usage. For this purpose, treatment planning computed tomography scans of thirty-three consecutive early stage breast cancer patients following breast conservation surgery were analyzed. After delineation of the breast clinical target volume (CTV) and placing opposed wedge paired isocenteric tangential portals, dosimeteric calculations were conducted and dose volume histograms (DVHs) were generated, first with pure $6 \mathrm{MV}$ photons and then these calculations were repeated ten times with incorporating $18 \mathrm{MV}$ photons (ten percent increase in weight per step) in each individual patient. For each calculation two indexes including maximum dose in the breast CTV $\left(\mathrm{D}_{\text {max }}\right)$ and the volume of $\mathrm{CTV}$ which covered with $95 \%$ Isodose line $\left(\mathrm{V}_{\mathrm{CTV}, 95 \% \mathrm{IDL}}\right)$ were measured according to the DVH data and then normalized values were plotted in a graph. The optimal weight of $18 \mathrm{MV}$ photons was defined as the intersection point of $\mathrm{D}_{\max }$ and $\mathrm{V}_{\mathrm{CTV}, 95 \% \text { IDL }}$ graphs. For creating a model to predict this optimal weight multiple linear regression analysis was used based on some of the breast and tangential field parameters.The best fitting model for prediction of $18 \mathrm{MV}$ photons optimal weight in breast radiotherapy using mixed energy technique, incorporated chest wall separation plus central lung distance (Adjusted R2=0.776). In conclusion, this study represents a model for the estimation of optimal beam weighting in breast radiotherapy using mixed photon energy technique for routine day to day clinical usage.
\end{abstract}

Keywords: Breast neoplasms - radiotherapy - treatment planning - high energy photons - toxicity

Asian Pac J Cancer Prev, 16 (17), 7785-7788

\section{Introduction}

Breast conservation surgery (BCS) followed by whole breast irradiation (WBI) is the standard of care for early stage breast cancer. Numerous large scale well designed randomized clinical trials indicate that this treatment is comparable to mastectomy in terms of local tumor control and overall survival while it maintains patients cosmesis. Conventionally, low energy megavoltage ( $\leq 6 \mathrm{MV}$ ) opposed wedge-based tangential photon beams are used for breast radiotherapy. The objectives are covering the breast with a therapeutic homogenous dose distribution while protecting healthy tissues from excessive irradiation and toxicity (Edward C. Halperin, 2013).

It is well known that acute and late complications of WBI such as erythema, edema, desquamation, pain, and telangiectasia and breast hardness are related to heterogeneous dose distribution in target volume and limiting areas of breast from receiving excessive dose (radiation hot spots) is of particular importance for achieving an acceptable long term cosmesis (Taylor et al., 1995; Das et al., 1997; Carruthers et al., 1999; Stillie et al., 2011).

Due to the breast shape and variability in its contour, open or even wedge-based tangents are unable to create a homogenous dose distribution in all portions of the target volume, so many experts recommend to use intensitymodulated radiation therapy (IMRT) for elimination of these hot this complex and costly spots and therefore lowering treatment complications(Haffty et al., 2008; Pignol et al., 2008; Morganti et al., 2011; Smith et al., 2011). Unfortunately, due to limited resources, this costly technology is not widely available to many patients, particularly in developing countries (Grau et al., 2014).

Utilization of higher energy photons (10-18MV) is a practical way to reduce these radiation hot spots particularly in large breasts. The main disadvantage of this approach is under dosing of superficial subcutaneous 
tissues in the buildup region of the beams (Ellen et al., 1999; Baird et al., 2001; Lief et al., 2007) and so an important question is: what proportion of high energy photons should be used in radiotherapy of the intact breast to achieve acceptable hot spots and at the same time adequate target coverage?

This study tries to create a model for estimating the optimal weight of high energy photon based on breast and tangential field characteristics for incorporation in breast radiotherapy.

\section{Materials and Methods}

\section{Study design}

Treatment planning computed tomography (CT) images of thirty three consecutive early stage breast cancer patients who were referred for WBI after BCS to our institution (Clinical Oncology Department of Golestan Hospital, Ahwaz Jundishapur University of Medical Sciences, Ahvaz, Iran) from May to November 2014 were evaluated in this study. Because confidentiality of the patients was not compromised, institutional ethics committee approval was not deemed necessary.

\section{Treatment planning}

For CT planning, patients were positioned supine on a breast board to make the chest wall slope parallel to the table couch with arms abducted and externally rotated. Palpable breast tissue, tangential field borders (medial border: mid-sternal line, lateral border: $2 \mathrm{~cm}$ beyond all palpable breast tissue or mid-axillary line, inferior border: $2 \mathrm{~cm}$ from infra-mammary fold and superior border: head of clavicle or second intercostal space) and surgical scars were marked with radiopaque wires and then axial thin CT slices were obtained from the mid neck to the upper abdomen(Edward C. Halperin, 2013). Ultimately patient data was transferred to the treatment planning software (Isogray v4.3, Dosisoft) for target volume delineation and beam positioning. After outlining breast clinical target volume (CTV) according to the RTOG protocol (http://www.rtog.org/CoreLab/ ContouringAtlases/BreastCancerAtlas.aspx), opposed wedge paired (15 degree, physical type) isocenteric tangential portals were generated according to breast CTV and previously mentioned anatomic landmarks without regional lymphatic irradiation. Dosimeteric calculations (with collapsed cone algorithm) were done with pure 6MV photons and then repeated ten times with incorporating $18 \mathrm{mv}$ photons (ten percent increase in weight per step) in each individual patient. This means that the last calculation was done with only $18 \mathrm{MV}$ photons. Prescription point was placed at the isocenter (approximately in the middle of the breast tissue) and for measuring conformity indexes, dose volume histograms (DVH) were analyzed.

\section{$18 M V$ photons optimal weight measurement}

For this purpose, two indexes including maximum dose in the breast CTV $\left(\mathrm{D}_{\max }\right)$ and the volume of CTV that receives more or equal to $95 \%$ of the prescription dose $\left(\mathrm{V}_{\mathrm{CTV}, 95 \% \mathrm{IDL}}\right)$ (Isodose line) were measured according to the DVH data. After normalization to the best value in each

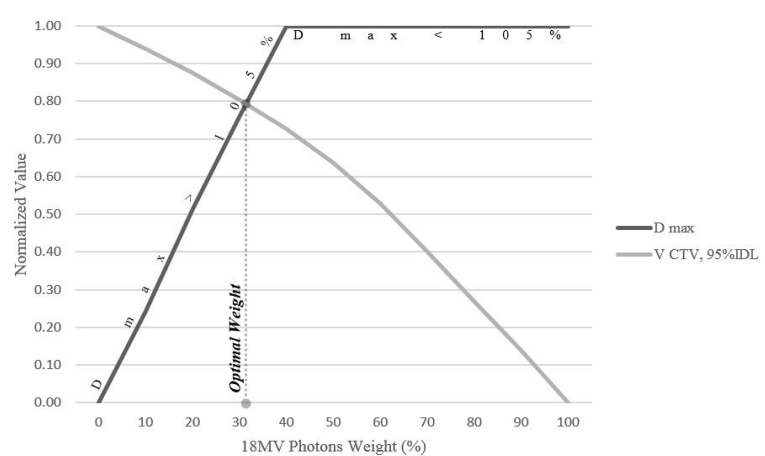

Figure 1. An example of Normalized $D_{\text {max }}$ and $V_{\text {CTV, }}$ 95\% IDL Values according to 18MV Photons Weight

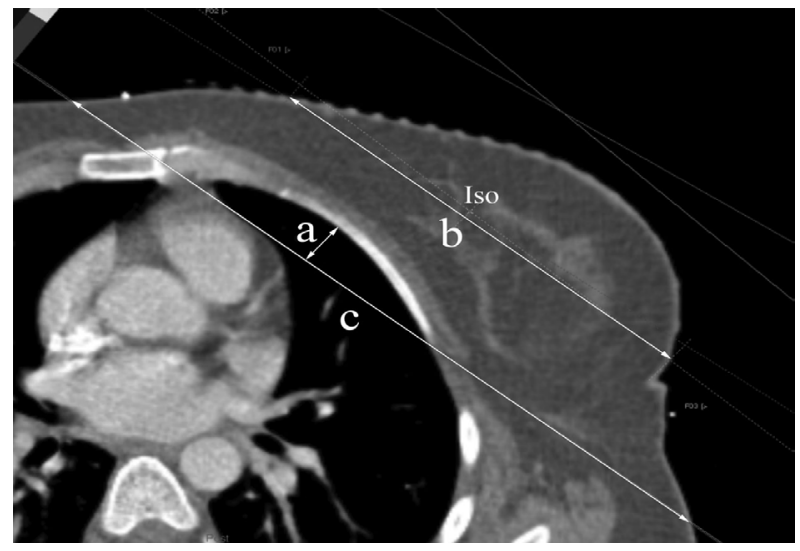

Figure 2. Central Axis in Treatment Planning CT Illustrating Definitions of CLD (a), SEP1 (b) and SEP2 (c)

patient (between 0-1), $\mathrm{D}_{\max }$ and $\mathrm{V}_{\mathrm{CTV}, 95 \% \text { IDL }}$ plotted in a single graph. Maximum CTV doses less than $105 \%$ were considered to be satisfactory. Then the optimal weight of $18 \mathrm{MV}$ photons in each individual was defined as the intersection point of $\mathrm{D}_{\max }$ and $\mathrm{V}_{\mathrm{CTV}, 95 \% \text { IDL }}$ graphs (Figure 1).

\section{$18 M V$ photons optimal weight prediction and Statistical} analysis:

For this purpose multiple linear regression analysis (forward stepwise method) was used to create a model based on parameters including breast CTV volume and tangential field's properties such as field height (Y), field width (X), central lung distance (CLD) and chest wall separations (SEP 1 and 2). These variables were checked for co-linearity with bivariate analysis (Pearson correlation). CLD, SEP1 and SEP2 were measured at the central axis as outlined in figure 2. Data was analyzed with SPSS version 13.0.

\section{Results}

Table 1 shows volume of CTV, tangential field's parameters used for WBI and measured optimal weight of $18 \mathrm{MV}$ photons in this patient population.

The best fitting model for prediction of $18 \mathrm{MV}$ photons optimal weight (Adjusted R2=0.776), incorporated SEP1, SEP2 and CLD as shown in the following and table 2:

$18 \mathrm{MV}$ photons optimal weight $(\%)=6.2 \times \mathrm{SEP} 1+$ $8.82 \times$ CLD $-2.23 \times$ SEP $2-25.18$ 
Table 1. Breast CTV volume, Tangential Fields Characteristics and Measured Optimal Weight of 18MV Photons

\begin{tabular}{lcr}
\hline Characteristic (Unit) & Median & Range \\
CTV VOL. (cm3) & 801 & $197-1951$ \\
Y $(\mathrm{cm})$ & 18 & $16-22$ \\
X $(\mathrm{cm})$ & 9.7 & $5.9-13$ \\
CLD $(\mathrm{cm})$ & 2.5 & $0.2-3.8$ \\
SEP 1 $(\mathrm{cm})$ & 15.6 & $9.9-20.3$ \\
SEP 2(cm) & 23.7 & $16.8-29$ \\
OPT. X18 $(\%)$ & 40 & $0-60$
\end{tabular}

CTV VOL. Breast CTV Volume, Y Tangential Field Height, X Tangential Field Width, CLD Central Lung Distance, SEP Chest Wall Separation, OPT. X18 Optimal Weight of 18MV Photons

Table 2. The Details of Best Fitting Regression Model

\begin{tabular}{|c|c|c|c|c|c|c|}
\hline Variable & Coefficient & SE & Importa & e $95^{\circ}$ & $\mathrm{CI}$ & P-value \\
\hline (Unit) & & & & Lower & Upper & \\
\hline & & 0.7 & & 4.77 & 7.64 & 0 \\
\hline m) & 8.82 & 1.52 & 0.23 & 5.7 & 11.94 & 0 \\
\hline $\mathrm{EP} 2(\mathrm{~cm})$ & -2.23 & 0.59 & 0.11 & -3.44 & -1.02 & 0.00 \\
\hline Constant & -25.18 & 12.73 & & -51.22 & 0.86 & 0.05 \\
\hline
\end{tabular}

SE Standard Error, CI Confidence Interval, SEP Chest Wall Separation, CLD Central Lung Distance

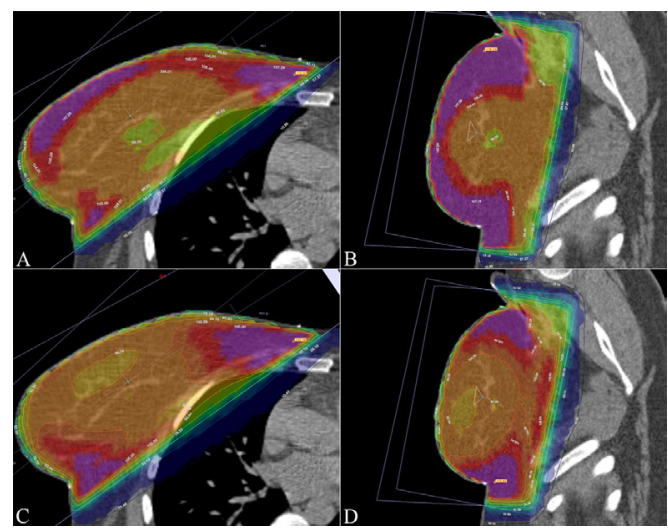

Figure 3. Isodose Lines in WBI without $(A, B)$ and with (C, D) 15 Degree Wedges. 105\% IDL is shown in red. Note significant hot areas in cephalic, caudal, medial and lateral portions of the breast despite using wedges

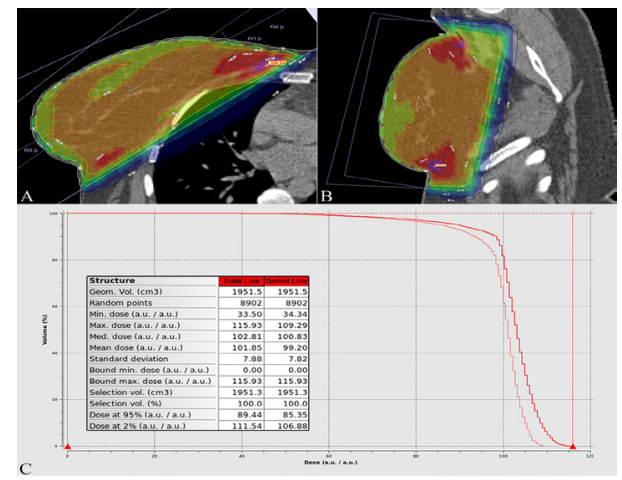

Figure 4. Incorporation of 18MV Photons with 55\% Weight (Measured Optimal Weight) in the same Patient Shown in figure 3 with Significant Reduction in Volume of $105 \%$ IDL (red) $(\mathbf{A}, \mathbf{B})$. Breast CTV DVH with pure 6MV (solid line) and mixed energy (dotted line) plans, shows remarkable decrease in severity and volume of hot areas and at the same time minimal underdosage of the target volume (C)

\section{Discussion}

Low energy x-rays (4-6 MV) are preferred for WBI. Commonly, conventional wedges are utilized to achieve a uniform dose distribution. But this happens only in the central axis (with elimination of sub-areolar hot regions) and significant inhomogeneity remain in other areas of the breast (Buchholz et al., 1997; Carruthers et al., 1999) (Figure 3). A reasonable approach to decrease the intensity and volume of these hot regions and also the breast integral dose is to use high energy photons ( $>6 \mathrm{MV}$ ) with a mixed energy technique (Figure 4).

With respect to the model we described above patients with higher SEP1 and CLD in contrast to SEP2, have hotter areas in the breast and need more high energy photons for a more homogenous treatment plan. Since hot regions in medial and lateral portions of the breast are due to a phenomenon called "lateral tissue effect"(Faiz M. Khan, 2014), increase in SEP1 and therefore prescription point depth intensifies this effect. Longer SEP2 means that photons entering the basilar portion of the breast transverse a longer path in the tissue and thus encounter more attenuation, so this has a favorable effect on hot areas in these regions. Central lung distance correlates with the lung volume in the tangential fields (Bornstein et al., 1990) so more CLD means less beam attenuation in the basilar portions of the breast (due to less absorption in the air) and thus more severe hot areas in medial and lateral sides of the breast.

This model is only validated when WBI is carried out with 15 degree wedge paired tangents using mixed 6 and $18 \mathrm{MV}$ photons. Other limitation of this dosimeteric study was that clinical outcomes such as acute and late toxicity as well as local tumor control were not assessed. We suggest that well designed clinical trials could be helpful to evaluate that if this possible dosimeteric advantage gained by incorporating $18 \mathrm{MV}$ photons in WBI with a weight calculated by this equation could translate to a more favorable clinical outcome.

In conclusion this study represents a model for estimation of optimal beam weighting in breast radiotherapy using mixed photon energy technique based on patient and tangential field parameters such as chest wall separations and central lung distance for day to day clinical usage

\section{Acknowledgements}

This study was Funded by the research and technology deputy of Ahvaz Jundishapur University of Medical Sciences, Ahvaz, Iran (Grant number: U92105).

\section{References}

Baird CT, Starkschall G, Liu HH, et al (2001). Verification of tangential breast treatment dose calculations in a commercial 3D treatment planning system.J Applied Clin Med Physics, 2, 73-84.

Bornstein BA, Cheng CW, Rhodes LM, et al (1990). Can simulation measurements be used to predict the irradiated lung volume in the tangential fields in patients treated for 
Buchholz TA, Gurgoze E, Bice WS, et al (1997). Dosimetric analysis of intact breast irradiation in off-axis planes. Int $J$ Radiat Oncol Biol Phys, 39, 261-7.

Carruthers LJ, Redpath AT, Kunkler IH (1999). The use of compensators to optimise the three dimensional dose distribution in radiotherapy of the intact breast. Radiother Oncol, 50, 291-300.

Das IJ, Cheng CW, Fein DA, et al (1997). Patterns of dose variability in radiation prescription of breast cancer. Radiother Oncol, 44, 83-9.

Edward C. Halperin DEW, Carlos A. Perez, Luther W. Brady 2013. Principles and practice of radiation oncology, Philadelphia, LWW.

Ellen MM, Hogstrom KR, Miller LA, et al (1999). A comparison of $18-\mathrm{MV}$ and $6-\mathrm{MV}$ treatment plans using 3D dose calculation with and without heterogeneity correction. Medical Dosimetry, 24, 287-94.

Faiz M. Khan JPG 2014. Khan's The Physics of Radiation Therapy, Philadelphia, LWW.

Grau C, Defourny N, Malicki J, et al (2014). Radiotherapy equipment and departments in the European countries: final results from the ESTRO-HERO survey. Radiother Oncol, 112, 155-64.

Haffty BG, Buchholz TA, McCormick B (2008). Should intensity-modulated radiation therapy be the standard of care in the conservatively managed breast cancer patient? $J$ Clin Oncol, 26, 2072-4.

Lief EP, Hunt MA, Hong LX, et al (2007). Radiation therapy of large intact breasts using a beam spoiler or photons with mixed energies. Med Dosim, 32, 246-53.

Morganti AG, Cilla S, de Gaetano A, et al (2011). Forward planned intensity modulated radiotherapy (IMRT) for whole breast postoperative radiotherapy. Is it useful? When? $J$ Applied Clin Med Physics, 12.

Pignol JP, Olivotto I, Rakovitch E, et al (2008). A multicenter randomized trial of breast intensity-modulated radiation therapy to reduce acute radiation dermatitis. J Clin Oncol, 26, 2085-92.

Smith BD, Pan IW, Shih YCT, et al (2011). Adoption of Intensity-Modulated Radiation Therapy for Breast Cancer in the United States. JNCI Journal of the National Cancer Institute, 103, 798-809.

Stillie AL, Kron T, Herschtal A, et al (2011). Does inverse planned intensity modulated radiation therapy have a role in the treatment of patients with left sided breast cancer? $J$ Med Imaging Radiation Oncol, 55, 311-9.

Taylor ME, Perez CA, Halverson KJ, et al (1995). Factors influencing cosmetic results after conservation therapy for breast cancer. Intern J Radiation Oncol* Biol* Physics, 31, 753-64. 